\title{
Praktische Umsetzung von gendersensibler Bildung für nachhaltige Entwicklung im GW-Unterricht am Beispiel "Nachhaltiger Konsum"
}

\author{
“ julia.wlasak@uni-graz.at, Regionales Zentrum für Expertise für Bildung für nachhaltige Entwicklung Graz-Styria, Karl-Franzens-Univer- \\ sität Graz \\ “" petra.wlasak@uni-graz.at, Regionales Zentrum für Expertise für Bildung für nachhaltige Entwicklung Graz-Styria, Karl-Franzens-Uni- \\ versität Graz
}

eingereicht am: 29.05.2017, akzeptiert am: 23.10.2017

\begin{abstract}
Bildung für nachhaltige Entwicklung (BNE) zielt darauf ab, Menschen unabhängig von ihrem Geschlecht in ihren diversen Lebensrealitäten gleichermaßen zu befähigen sich an der Gestaltung einer zukunftsfähigen Gesellschaft zu beteiligen. Dieser Beitrag stellt die GW-Unterrichtseinheit „Nachhaltiger Konsum“ vor, in welcher didaktische Grundsätze einer gendersensiblen Lehre und BNE angewandt werden. Die Unterrichtseinheit wurde im Rahmen einer 9. Schulstufe in einem Bundesgymnasium getestet und entsprechend den Bedürfnissen einer heterogenen Klasse mit Schüler/-innen unterschiedlichsten Erstsprachen, unterschiedlicher Herkunft und sozialem Hintergrund aufbereitet.
\end{abstract}

Keywords: Bildung für nachhaltige Entwicklung, Gender, gendersensible Didaktik, Diversität, Handlungsorientierung

\section{Gender sensitive education for sustainable development in practice by the example of "sustainable consumption"}

The aim of Education for Sustainable Development is to empower all human beings regardless of their gender and with regards to their diverse life-realities to participate in the creation of a sustainable future. This article presents the Geography and Economic teaching unit "Sustainable Consumption", in which didactic principles of gender-sensitive teaching and ESD are applied. The teaching unit was tested in a $9^{\text {th }}$ grade of a federal grammar school and adapted to a highly diverse class with of pupils with various first languages, origins and social backgrounds.

Keywords: Education for Sustainable Development, gender, gender-sensitive teaching, diversity, action-orientation

\section{$1 \quad$ Einleitung}

Die Inklusion einer kritischen Genderperspektive bei der Erschließung von Bildung für nachhaltige Entwicklung (BNE) im Rahmen des Geographie- und Wirtschaftskunde (GW) Unterrichts ist essentiell, um Schüler/innen ein Bewusstsein für Geschlechterungleichheit in unterschiedlichsten und intersektionalen Ausprägungen und als mögliche Ursache nichtnachhaltiger Werte, Denk- und Verhaltensweisen zu vermitteln. Auf diese Weise kann das Ziel der Darstellung eines ganzheitlichen Ansatz für das Verständnis von komplexen Zusammenhängen und die Notwendigkeit der Kritik an bestehenden Ungleichheiten in einer globalisierten Welt verständlich gemacht werden und darauf basierend „selbstständiges Erkennen von Problemen und das Finden von Wegen zu ihrer Lösung" (Bundesministerium für Bildung 2016: 60) mitgegeben werden. Wie eine kritische Genderperspektive dafür sensibilisieren kann, dass es sich bei weltweiten ungleichen und nicht-nachhaltigen Verhältnissen um vergeschlechtlichte Strukturen handelt, die Menschen trotz ihrer Diversität ungleiche, auf der Norm der Zweigeschlechtlichkeit und der Heteronormativität beruhende sowie auf Stereotype basierende soziale Rollen und Positionen zuteilen, wird in diesem Heft von GW-Unterricht im Fachdidaktik-Beitrag ,Perspektivenerweiterung durch kritische Gender 
Studies: Gendersensible Bildung für nachhaltige Entwicklung im GW-Unterricht' im Detail aufgezeigt.

Um den angestrebten ganzheitlichen Ansatz von BNE im GW-Unterricht zu realisieren, ist es notwendig, gendersensible didaktische Methoden anzuwenden, damit Geschlechterungleichheit als wesentliche Ursache einer nicht-nachhaltigen Entwicklungen erkannt, thematisiert und problematisiert wird.

Die Grundsätze einer gendersensiblen Didaktik sind im Grundsatzerlass zum Unterrichtsprinzip ,Erziehung zur Gleichstellung von Frauen und Männern ‘ des Bundesministeriums für Bildung vorgegeben (vgl. Bundesministerium für Bildung 2014; Bundesministerium für Bildung 2017). Umso erstaunlicher ist es, dass diese nicht explizit im GW-Lehrplan erwähnt werden und im GW-Lehrplan trotz des Bekenntnisses der Vermittlung von globaler Ungleichheit, Inklusions- und Exklusionsmechanismen sowie Diversität hierbei nicht auf Geschlechterverhältnisse und deren hierarchische binäre Ordnung eingegangen wird. Auch werden die Vermittlung von Intersexualität und damit die Konstruktion der Vorstellung von nur zwei existierenden Geschlechtern nicht thematisiert (vgl. Koyama \& Weasel 2003).

Das Verständnis scheint dafür gering, dass ungleiche Geschlechterverhältnisse, die Frauen in ihrer Vielfalt weltweit ungleiche Lebenschancen, Ressourcen und weniger Möglichkeiten für eine selbstbestimmte Entwicklung geben, ein Grund einer nicht-nachhaltigen Entwicklung sind (vgl. Bauhardt 2010: 325). Zwar gibt es ein Bekenntnis der internationalen Gemeinschaft und nationaler Regierungen, Genderungleichheiten in und durch Bildungsarbeit abzubauen. Ein umfassender Ansatz, welcher eine kritische Geschlechterperspektive in Bildung für nachhaltige Entwicklung global als auch national inkludiert, fehlt aber bisher (siehe für detaillierte Ausführungen den genannten Beitrag in der Rubrik Fachdidaktik).

Dieser Beitrag stellt eine Unterrichtseinheit vor, die zum Ziel hat, BNE im Rahmen des GW-Unterrichts gendersensibel umzusetzen. Dabei wird die Aufgabe des GW-Unterrichts, BNE zu fördern, an die Grundsätze des gendersensiblen Unterrichts gekoppelt, um eine gendersensible Umsetzung von BNE im GWUnterricht realisieren zu können und damit den ganzheitlichen Ansatz von BNE zu erfüllen.

\section{Grundsätze von BNE und gendersensibler Didaktik im GW-Unterricht}

BNE im Rahmen des GW-Unterrichts hat zum Ziel, Schüler/innen zu mündiger und aktiver Partizipation für die Gestaltung einer zukunftsfähigen Gesellschaft zu befähigen, zu ermutigen und anzuhalten (vgl. Frid- rich 2016: 24). Dies orientiert sich an dem normativen Grundsatz des Zieles einer inter- und intragenerationellen Gerechtigkeit (vgl. Zimmermann 2016b: 4), welche daran appelliert, allen jetzt und in Zukunft lebenden Menschen, unabhängig von Geschlecht, Alter, Herkunft, Wohnort, Bildungshintergrund, Besitz, Arbeitsverhältnis, Kultur, Religion, sexueller Orientierung, Familienstatus, Behinderung und Krankheit die gleichen Möglichkeiten und Ressourcen für die Gestaltung ihres Lebens garantieren zu können.

Bei der Erschließung von BNE ist es erforderlich, den Schülerinnen und Schülern den ganzheitlichen Ansatz einer nachhaltigen Entwicklung bewusst zu machen, welcher sowohl die ökonomische, ökologische als auch soziale und kulturelle Dimension inkludiert. Systemische Zusammenhänge, Ursachen und Auswirkungen von globalen Entwicklungen im räumlichen Kontext sollen so aufgezeigt werden. Dies inkludiert die Sensibilisierung für die Ursache und Auswirkungen ungleicher gesellschaftlicher Verhältnisse und Benachteiligungen und damit auch einen Blick auf ungleiche Geschlechterverhältnisse in ihrer Diversität. BNE sollte von partizipativen, handlungsorientierten und interaktiven Methoden geprägt sein, welche die lokale Lebenssituation der Schüler/innen berücksichtigen und einen Fokus auf Handlungsorientierung setzen (siehe hierzu auch die Prinzipien von BNE im Beitrag, Perspektivenerweiterung durch kritische Gender Studies: Gendersensible Bildung für nachhaltige Entwicklung im GW-Unterricht').

Bei der Gestaltung eines gendersensiblen Unterrichtssettings orientieren wir uns an den zehn Grundsätzen einer gendersensiblen Lehre, welche im genannten Artikel in der Rubrik Fachdidaktik ausgeführt wurden (vgl. Bundesministerium für Unterricht, Kunst und Kultur 2008).

Die hier aufgezählten Grundsätze gendersensibler Lehre werden im Rahmen der vorliegenden Unterrichtseinheit durchgehend inkludiert. Ziel dabei ist es außerdem, die Grundsätze von BNE zu inkludieren.

\section{Das Thema ,Nachhaltiger Konsum' in gendersensiblem Kontext}

Als Thema der Unterrichtseinheit wurde nachhaltiger Konsum gewählt. Eine Gesellschaft ist nur dann zukunftsfähig, wenn es ihren Mitgliedern bewusst ist, dass (1) übermäßiger Konsum Einfluss auf den weltweiten zu hohen Ressourcenverbrauch und die globale ungleiche Verteilung von bestehen Ressourcen hat und dass (2) eine eigene Verhaltensänderung hier entgegensteuern kann (vgl. Zimmermann 2016a: 26). Individuelle Konsumentscheidungen und -verhalten beeinflussen, ob umweltfreundlich, regional und fair 
(1) Bewusstseinsbildung und gemeinsame Dekonstruktion von Geschlechterstereotypen

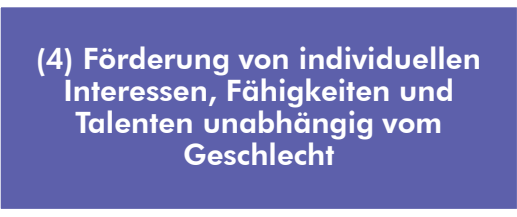

(7) Verständnis über die gemeinsamen Grundwerte eines gleichberechtigten,

wertschäłzenden Umgangs miteinander

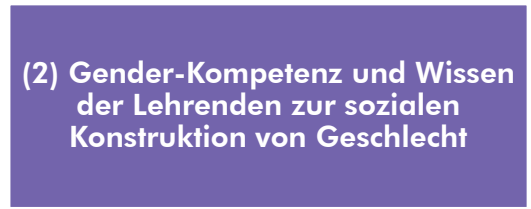

(5) Schaffung eines offenen, schützenden, inklusiven Lernklimas
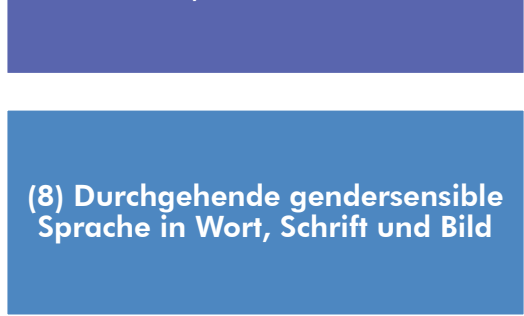

(10) Gender Mainstreaming auf institutioneller Ebene

(Lehrplan, Aus- und Fortbildung der Lehrenden, Bildungspolitik, Mädchen- und Jungenförderungsprogramme etc.)

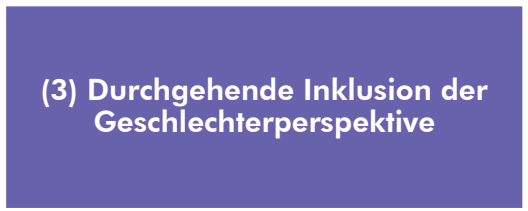

(6) Bewusste Förderung und Empowerment von Mädchen und Jungen um sie anzuregen, auf gleiche Weise aktiv mitzuwirken

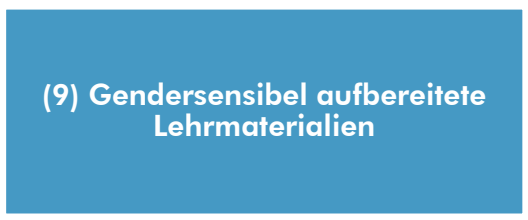

Abb. 1: Grundsätze gendersensibler Lehre (eigene Darstellung) gehandelte Konsumgüter nachgefragt werden oder nicht, und haben Einfluss auf den weltweiten Ressourcenverbrauch und die globale Verteilung von bestehenden Ressourcen (vgl. ebd.: 44). Der Kauf von Waren und Dienstleistungen ist auch in den Lebenswelten der Schüler/innen alltäglich präsent und kann daher konkret mit ihrem Alltag verbunden werden. So geht es in dieser Praxis im Rahmen der Kompetenzorientierung um die Entwicklung der Orientierungs-, Urteils- und Handlungsfähigkeit (vgl. Haarmann 2014: 208), um es Schülerinnen und Schülern zu ermöglichen, mündig, kompetent und werteorientiert in wirtschaftlich geprägten Lebenswelten zu handeln (vgl. Fridrich 2014: 17).

Nachhaltiger Konsum kann und muss auf lokaler Ebene auf vielfältige Weise umgesetzt werden. Die Behandlung dieses spezifischen Themas im Kontext von BNE erfordert eine Sensibilisierung dafür, dass Menschen auf dieser Welt unterschiedliche Lebensrealitäten vorfinden und demnach unterschiedliche Möglichkeiten für nachhaltige Konsumverhaltensweisen haben. Insbesondere verschiedene Geschlechter zeigen aufgrund ihrer ungleichen Arbeits-, Einkommens-, und Besitzverhältnisse unterschiedliche Konsummöglichkeiten und haben dementsprechend auch unterschiedliche Handlungsoptionen hinsichtlich der Gestaltung einer nachhaltigen Gesellschaft (vgl. Wastl-Walter 2010: 184ff.). Männer und Frauen in ihren vielfältigen und unterschiedlichen Lebenslagen weisen aufgrund von unterschiedlichen sozioökonomischen Ausgangspositionen und soziokultureller Prägung unterschiedliches Konsumverhalten auf, welches aber ebenso mit einer problematischen, historisch gewachsenen Zuschreibung von Stereotypen und Rollenbildern verbunden ist (vgl. Althans 2016). Diese Unterschiede und die daraus resultierenden Ungleichheiten basieren ebenso auf der Verknüpfung von Geschlecht mit anderen sozialen Kategorien wie beispielsweise Klasse, Hautfarbe, Geburtsort, Herkunft oder Alter (vgl. Winkler \& Degele 2009). Das bedeutet, dass beispielsweise eine weiße Frau aufgrund ihrer Privilegien andere Konsummöglichkeiten hat als eine schwarze Frau, beziehungsweise dass Frauen mit Migrationshintergrund in Österreich andere Formen der Diskriminierung am Arbeitsplatz erfahren als Frauen ohne Migrationshintergrund und dadurch auch limitierte Konsummöglichkeiten haben (vgl. Münst 2008).

Das Themengebiet ,Nachhaltiger Konsum' eignet sich demnach sehr gut, ungleiche Geschlechterverhältnisse zu thematisieren bei einem gleichzeitigen Fokus auf individuelle Handlungsorientierung. Es ist außerdem im Lehrplan der AHS für das Fach GW in der Sekundarstufe II in der 1. Klasse zu finden. So sollen sich Schüler/innen mit der, Produktion von Bedürfnissen hinsichtlich Konzepten der Nachhaltigkeit auseinandersetzen und diese bewerten sowie die ,Tragfähigkeit der Einen Welt zukunftsorientiert reflektieren` (vgl. Bundesministerium für Bildung 2016: 64).

\section{$4 \quad$ Lernziele der Unterrichtseinheit}

Das Basiskonzept ,Nachhaltigkeit und Lebensqualität im GW-Unterricht weist daraufhin, dass Nachhaltig- 
keit stark mit Lebensqualität verbunden ist. Lebensqualität sowie die damit einhergehenden individuellen Entfaltungsmöglichkeiten sollen allen Menschen auf dieser Einen Erde, zum jetzigen Zeitpunkt und auch zukünftig, geboten werden. Die Schüler/innen sollen laut dem Basiskonzept in der Praxis überlegen, wie ein Ausgleich des zerbrechlichen Mensch-Umwelt-Systems anhand nachhaltigen Konsums stattfinden kann und dennoch alle Menschen auf dieser Welt ihre Bedürfnisse befriedigen können. Dabei sollen sie sich Werte und Wissen aneignen, um einen Lebensstil zu erlernen und aktiv eine lebenswerte Zukunft für alle zu gestalten (vgl. Bundesministerium für Bildung 2016: 62). Die Schüler/innen sollen in Anlehnung an das Basiskonzept ,Arbeit, Produktion und Konsum erkennen, dass der Mensch täglich selbst ökonomisch tätig ist, indem er bzw. sie konsumiert. Dieser, in der Regel übermäßige, Konsum soll in der Praxis kritisch reflektiert werden und Handlungsmöglichkeiten gefunden werden, die Konsum aus einer nachhaltigen Perspektive behandeln (vgl. ebd.). Dabei geht es darum, dass die Schüler/innen die Komplexität ökonomisch geprägter Lebenswelten in ihrer Vielfalt erfassen. Neben Fachwissen und auf Fakten basierende ökonomische Denkweisen werden ebenso ethische Fragestellungen in diesem Zusammenhang aufgegriffen (vgl. Mehren et al. 2015).

Der Fokus liegt in diesem Beitrag auf eine gendergerechte Behandlung des Themas und die Einbeziehung unterschiedlicher Lebensarten auf dieser Welt. Mit der Umsetzung der Unterrichtseinheit sollen Schüler/ innen und Lehrer/innen befähigt werden, bestehende soziale Ungleichheiten zu erkennen und zu problematisieren sowie marginalisierte Personen(gruppen) zu ermächtigen, nachhaltiges Verhalten umzusetzen.

Das übergeordnete Ziel der vorliegenden Unterrichtseinheit ist es, dass Schüler/innen selbst aktiv werden und konkrete Handlungsmöglichkeiten zur fairen Gestaltung durch Diversität geprägte Lebenswelten beitragen, um gemeinsam auf eine gerechtere, nachhaltige Welt hinzuarbeiten, in der sich alle Individuen inkludiert fühlen können und inkludiert sind. Hierfür müssen sie erkennen, dass für eine nachhaltige Entwicklung konkret gesetzte Handlungen im eigenen Lebensumfeld notwendig sind und diese je nach individueller Lebenslage unterschiedlich ausfallen können.

Es soll begreifbar gemacht werden, dass Menschen unterschiedlichen Geschlechts auf dieser Welt unterschiedliche Lebensweisen und individuelle Bedürfnisse haben und es somit individueller Handlungsmöglichkeiten bezogen auf Konsum bedarf, um Lebensqualität ermöglichen zu können. Das Thema Konsum eignet sich somit für eine Bewusstseinsbildung für Geschlechterverhältnisse.
Schüler/innen sollen nachhaltige Entwicklung im Kontext ihrer Lebensrealitäten im Rahmen des Lehrplans für den GW-Unterricht erfahren und hierbei das Thema Geschlechtergerechtigkeit konsequent reflektieren. Hierfür werden die Prinzipien eines gendersensiblen Unterrichts eingehalten, die Schüler/innen ermutigt, soziale und intersektionale Ungleichheiten zu problematisieren, und ermächtigt, lösungsorientierte und zukunftsfähige Verhaltensweise zu erarbeiten und umzusetzen.

Lokale Betroffenheit und Verantwortungsbewusstsein sollen dabei entwickelt werden und damit das eigene Verhalten kritisch reflektiert und nachhaltig verändert werden. So soll ein Bewusstsein für die Diversität von Lebenssituationen geschaffen werden und für ein Verständnis, dass nicht alle Lösungen für alle Menschen auf Grund ihrer ungleichen Lebensumstände umsetzbar sind. Gleichzeitig soll hierbei die Botschaft vermittelt werden, dass jede und jeder in Anbetracht der jeweiligen Lebensumstände doch etwas dazu beitragen kann, eine nachhaltige Entwicklung zu realisieren. Damit wird auch das Bewusstsein dazu gestärkt, dass die Schüler/innen selbst auch, egal in welcher Lebenssituation sie sich befinden - familiär, ökonomisch, kultureller Hintergrund - auch etwas verändern und gestalten können, denn gesellschaftlicher Wandel muss als kommunikativer und materieller Prozess allen Individuen offenstehen.

Die Lernziele in dieser Praxis werden wie folgt formuliert:

- Die Schüler/innen können verstehen, dass die ökologischen Ressourcen begrenzt sind, dass es soziale und ökonomische Ungleichheiten gibt und die wirtschaftlichen Bedürfnisse des Menschen erkennen.

- Die Schüler/innen erkennen die Vielfalt lebensräumlicher Wirklichkeiten und nehmen sozioökonomische und sozio-kulturelle Prägungen wahr und können diese vergleichen.

- Die Schüler/innen erhalten Übung in der Anwendung einer gendergerechten Sprache.

- Die Schüler/innen können Geschlechterungleichheiten benennen und problematisieren.

- Die Schüler/innen können Stereotype reflektieren und dekonstruieren.

- Die Schüler/innen können Handlungsoptionen in den entsprechenden Rollen erkennen und reproduzieren.

- Die Schüler/innen können konkrete Lösungsoptionen eigenständig kritisch beurteilen.

- Die Schüler/innen bilden Verantwortungsbewusstsein für einen nachhaltigen Lebensstil in unterschiedlichen Lebenslagen. 


\section{Vorbereitung und Ablauf der Unterrichts- einheit}

Als Vorbereitung für die vorliegende Unterrichtseinheit lesen die Schüler/innen bereits in der vorhergehenden Unterrichtsstunde einen Zeitungsartikel von Eder-Kornfeld (2014), der den Einstieg zum Thema erleichtern soll. Bei dem Text handelt es sich um eine Reportage, die unterschiedliche Projekte und Aktionen von Einzelpersonen beschreibt, welche sich zum Ziel gesetzt haben, einen nachhaltigen Konsum- und Lebensstil zu betreiben. Da dieser Text nicht in einer gendergerechten Sprache formuliert ist und auch keine genderspezifischen Aspekte behandelt werden, sollte dies bereits in der Vorbereitung durch die Lehrperson angesprochen und mit den Schülerinnen und Schülern diskutiert worden sein.

Vor Beginn der Unterrichtseinheit besinnt sich die Lehrperson auf die Prinzipien eines gendersensiblen Unterrichts, um diese konsequent im Unterricht zu inkludieren. Dementsprechende Genderkompetenz ist hierfür Voraussetzung. Eine gendergerechte Sprache umrahmt die Einheit, auf Nichtbeachtung dieser muss explizit hingewiesen werden. Lehrmaterial muss dementsprechend vor- und aufbereitet werden. Der Raum, in dem die Einheit stattfindet, muss eine barrierefreie Infrastruktur für alle vorweisen, ein respektvolles Miteinander und eine vertrauensvolle Umgebung während der Einheit bilden unerlässliche Elemente. Ziel dabei ist es, einen Raum für ein offenes, schützendes und inklusives Lernklima zu schaffen.

Als Einstieg für die Unterrichtseinheit wird eine Präsentation zum Thema ,Nachhaltiger Konsum' gezeigt und dazu der gelesene Zeitungstext im Plenum aufgegriffen, um je nach Wissensstand der jeweiligen Klasse bereits Gelerntes zu wiederholen bzw. neues Wissen zu generieren und dieses zu reflektieren. Es ist wichtig, hierbei erneut gendersensible Themen wie gendergerechte Sprache des Textes und Rollenverteilungen der im Text beschriebenen Personen anzusprechen und somit die bewusste Einbindung von Mädchen und Jungen anzuregen, damit diese auf gleiche Weise aktiv mitwirken.

Nach dem Einstieg beginnt der Hauptteil der Einheit, in welcher ein Rollenspiel und die Methode ,Heißer Stuhl' (vgl. Lackner 2012) angewandt werden.

Für das Rollenspiel gibt es insgesamt vier unterschiedliche Personenbeschreibungen (siehe Tab.1 und im Detail bei den Unterrichtsmaterialien als Kopiervorlage).

Die Rollenbeschreibungen zeichnen sich dadurch aus, dass die darin beschriebenen Personen eine hohe Diversität hinsichtlich Herkunft, Alter, Familienkonstellation, sexueller Orientierung und sozio-ökonomischer Position aufweisen und keine stereotypen
Tab. 1: Übersicht Personenbeschreibungen für das Rollenspiel

\begin{tabular}{|c|c|c|c|c|}
\hline Rolle & 1 & 2 & 3 & 4 \\
\hline Name & Amir & Lisa & Peter & Sarah \\
\hline Alter & 17 & 20 & 39 & 55 \\
\hline $\begin{array}{l}\text { Lebens- } \\
\text { situation }\end{array}$ & $\begin{array}{l}\text { unbegleiteter } \\
\text { Asylwerber }\end{array}$ & $\begin{array}{l}\text { in Partner- } \\
\text { schaft mit } \\
\text { einer gleich- } \\
\text { altrigen Frau, } \\
\text { allein lebend }\end{array}$ & $\begin{array}{l}\text { alleinerzie- } \\
\text { hender Vater } \\
\text { von zwei } \\
\text { Kindern }\end{array}$ & $\begin{array}{l}\text { in einer } \\
\text { Partnerschaft } \\
\text { mit jüngerem } \\
\text { Mann lebend }\end{array}$ \\
\hline $\begin{array}{l}\text { Wohn- } \\
\text { form }\end{array}$ & $\begin{array}{l}\text { Flüchtlings- } \\
\text { quartier ohne } \\
\text { Verpflegung }\end{array}$ & $\begin{array}{l}\text { Studierenden- } \\
\text { Wohngemein- } \\
\text { schaft }\end{array}$ & Mietwohnung & $\begin{array}{l}\text { Eigentums- } \\
\text { wohnung }\end{array}$ \\
\hline Beruf & $\begin{array}{l}\text { Schüler der } \\
\text { externen } \\
\text { Hauptschule }\end{array}$ & $\begin{array}{l}\text { Studentin der } \\
\text { technischen } \\
\text { Chemie }\end{array}$ & Angestellter & $\begin{array}{l}\text { Geschäftsin- } \\
\text { haberin eines } \\
\text { Computer- } \\
\text { fachgeschäfts }\end{array}$ \\
\hline $\begin{array}{l}\text { Einkom- } \\
\text { men }\end{array}$ & $\begin{array}{l}150 \text { Euro } \\
\text { pro Monat }\end{array}$ & $\begin{array}{l}680 \text { Euro } \\
\text { Stipendium } \\
\text { pro Monat }\end{array}$ & $\begin{array}{l}1650 \text { Euro } \\
\text { netto pro } \\
\text { Monat }\end{array}$ & $\begin{array}{l}4000 \text { Euro } \\
\text { pro Monat }\end{array}$ \\
\hline
\end{tabular}

Annahmen über Frauen oder Männer beinhalten. Ziel hierbei ist es, dass die Schüler/innen angehalten werden, vorhandene stereotype Denkmuster durch diese Übung aufzubrechen, alternative Lebensweisen kennenlernen und sich in diese hineindenken lernen. Zusätzlich sehen die Schüler/innen ihre eigene Diversität in den Rollenbeschreibungen repräsentiert und nehmen diese dadurch als anerkannt wahr.

Die Methode des Rollenspiels bietet außerdem den Schülerinnen und Schülern einen geschützten Rahmen, um alternative Perspektiven nach außen hin zu vertreten, da hierbei offiziell keine persönliche Position vertreten wird.

Die einzelnen Rollenbeschreibungen werden den Schülerinnen und Schülern ausgehändigt, wobei jeder Schülerin und jedem Schüler eine Rolle zugewiesen wird. Die Rollen sollen dabei in gleicher Anzahl im Klassenverband vorkommen. Anschließend werden die Schüler/innen in Vierergruppen Sitzkreisen zugeteilt. In jedem Vierer-Sitzkreis muss jeweils eine Vertretung pro Personenbeschreibung sitzen.

Die Schüler/innen haben nach der Zuteilung zehn Minuten Zeit, ihre zugewiesene Rolle durchzulesen und sich hineinzudenken und zu -fühlen. Die anschließende Aufgabenstellung dazu lautet, sich in die jeweilige Rolle hineinzuversetzen und im Anschluss den Lebensstil, Tagesablauf und diverse (angenommene) Charakteristika der Rolle zu beschreiben und sich zu überlegen, wie diese Personen nachhaltigen Konsum praktizierten könnte (siehe bei den Unterrichtsmaterialien die Aufgabenstellung als Kopiervorlage).

Die Rolle, die eingenommen wird, ermöglicht einen Perspektivenwechsel, der es auch schüchternen Teilnehmenden erlaubt, an der Diskussion teilzunehmen, indem ein Standpunkt vertreten wird, der nicht 
Tab. 2: Überblick Unterrichtseinheit, Nachhaltiger Konsum ‘

\begin{tabular}{ll}
\hline Thema & Nachhaltiger Konsum auf lokaler Ebene \\
Dauer & 50 Minuten \\
Gruppengröße & Rund 20 Schüler/innen \\
Methoden & Vortrag, Rollenspiel, Heißer Stuhl, gemeinsame Abschlussreflexion \\
\hline Benötigte Materialien & - Einstiegspräsentation \\
& - Ausgedruckte Rollenbeschreibungen jeweils in Anzahl eines Viertels der gesamten Teilneh- \\
& mer/innenzahl \\
& - Flipchart oder Tafel mit Schreibutensilien (Stifte oder Kreide) \\
\hline Zeitlicher Überblick & - Einstiegspräsentation und Gruppeneinteilung: 10 Minuten \\
& - Individuelles Einlesen in den Text: 10 Minuten \\
& - Heißer Stuhl: 4x5 Minuten: 20 Minuten \\
& - Abschlussreflexion: 10 Minuten \\
\hline Raumgestaltung & - Plenum für Einstiegspräsentation und Abschlussreflexion \\
& - Tischinseln mit je 4 Sesseln für Rollenspiel mit Gruppenarbeiten \\
\hline
\end{tabular}

der eigene ist. Dadurch wird eine angenehme und schützende Atmosphäre erzeugt bzw. verstärkt und die Erfahrung eines gleichberechtigten, wertschätzenden Umgangs miteinander ermöglicht.

Anschließend beginnt die Methode, Heißer Stubl: Dabei gilt es sich in die erhaltene Rolle zu versetzen und sich so den Fragen einer Gruppe zu stellen:

Die erste Runde des ,Heißen Stuhls' beginnt und Rolle 1 eines jeden Sitzkreises stellt sich der Gruppe in der jeweiligen Rolle vor. Die Gruppenmitglieder beginnen mit Intensivinterviews und stellen Fragen an Rolle 1 bezogen auf Konsumverhalten, Einstellungen, Werte und Handlungsoptionen. Die Fragen werden rollenkonform von der Person auf dem ,Heißen Stuhl beantwortet. Leitfragen für diese Interviews werden zuvor an die Schüler/innen ausgeteilt, um angeregte Interviews zu ermöglichen. Die Leitfragen finden sich als Kopiervorlagen bei den Unterrichtsmaterialien, können aber auch im Vorfeld gemeinsam mit den Schülerinnen und Schülern, beispielsweise in einer Vorbereitungsstunde zum Thema Nachhaltigkeit, erarbeitet werden. Sind die Schüler/innen bereits gut über das Thema Nachhaltigkeit informiert, können die Fragen auch spontan von den Schülerinnen und Schülern entwickelt werden. Sind die Fragen beantwortet bzw. werden keine Fragen mehr gestellt, wechselt Rolle 2 auf den ,Heißen Stuhl: Die Lehrenden beobachten währenddessen die Arbeit der Gruppen und leisten Hilfestellung bei Fragen. Nachdem sich alle Rollen auf dem ,Heißen Stuhl' behauptet haben, rückt die Klasse wieder im Plenum zusammen.

In der abschließenden Reflexion werden die Lösungsansätze der einzelnen Rollen zusammengetragen und reflektiert. Sie werden schriftlich an der Tafel festgehalten. Fragen und Diskussion sind willkommen. Die Lehrperson ist dabei aufgefordert, die von den Schülerinnen und Schülern präsentierten Lösungen anzuhalten zu reflektieren, ob die verschiedenen Lösungsansätze für mehrere Personen in Frage kommen und auf soziale und kulturelle Unterschiede in den Rollen hinzuweisen, um zu vermeiden, dass hauptsächlich ökonomische Unterschiede in den Rollen genannt werden. Auch gilt es dabei, die Lösungsansätze in den Alltag der Schüler/innen zu integrieren. Die Schüler/innen sollen sich nun wieder von ihrer Rolle lösen und reflektierend die Lösungsansätze und Handlungsmöglichkeiten auf sich selbst übertragen. Eine weiterführende Aufgabe ist es, anhand eines Tagebuches das eigene Konsumverhalten zu dokumentieren und darüber zu reflektieren, um zu sehen, ob die ausgehandelten Lösungswege auch aktiv umgesetzt werden und somit den Weg in die eigene Lebenswelt finden.

Tabelle 2 gibt einen Überblick über die Unterrichtseinheit.

\section{Testung der Unterrichtseinheit: Gender- sensible BNE trifft Diversität}

\section{Die Testklasse}

Die Unterrichtseinheit wurde im November 2016 im Rahmen einer regulären GW-Einheit an einer 9. Schulstufe in einem Bundesgymnasium mit 19 Schülerinnen und Schülern, hiervon ca. 60\% Mädchen und $40 \%$ Burschen, in Graz von den zwei Autorinnen im Beisein des Klassenlehrers getestet ${ }^{1}$. Die Unterrichtseinheit wurde durch eine über der

Die Unterrichtseinheit wurde am 18.11.2016 von 11:35 bis 12:25 Uhr an der 5PK-Klasse im Bundesgymnasium Dreihackengasse in Graz mit freundlicher Unterstützung durch Martin Möderl getestet. 
Tafel installierten Videokamera aufgezeichnet. Dieses Material diente den zwei Autorinnen im Anschluss als Basis für die gemeinsame Reflexion und Auswertung der Testung ${ }^{2}$.

Bei der ausgewählten Testklasse handelt es sich um eine Gruppe von Schülerinnen und Schülern mit diversen kulturellen Hintergründen, Religionen, Hautfarben, unterschiedlichen Deutschkenntnissen und einer hohen Anzahl an unterschiedlichen Erstsprachen. Auch der familiäre und ökonomische Hintergrund der Schüler/innen ist sehr breit gefächert. So entstammen die Schüler/innen Familien mit diversen sozialen Konstellationen, sei es Großfamilien, Alleinerzieherinnen-Modellen und Patchworkfamilien (vgl. Hörmann et al. 2017). Mehrere Schüler/innen haben einen Flucht- oder Migrationshintergrund. Bildungshintergrund und Berufshintergrund der Eltern der Schüler/innen variieren ebenso ${ }^{3}$.

\section{Vorbereitungen}

Die kulturelle und sprachliche Diversität der Klasse verlangte umfassende Vorbereitungen von Seiten der Lehrenden, um eventuelle sprachliche Barrieren überwinden bzw. alternative Lehr- und Lernmethoden anwenden zu können und trotz der vorhandenen Vielfalt ein offenes und schützendes Lernklima für alle zu schaffen. So wurden die Begriffe Nachhaltigkeit, Wirtschaft, Natur, Soziales und Kultur im Vorfeld auf Englisch, Ungarisch und Arabisch übersetzt, um wesentliche Begriffe der Einheit in den mehrheitlich gebrauchten Nicht-deutschen-Erstsprachen der Schüler/ innen anführen zu können. Außerdem wurde ein Arbeitsblatt zu nachhaltigem Konsumverhalten auf Ungarisch für einen Schüler vorbereitet, welcher zum Zeitpunkt der Testung erst seit zwei Monaten in Österreich lebte und kaum über Deutschkenntnisse verfügte ${ }^{4}$.

Der Klassenlehrer hatte in den zwei Unterrichtseinheiten zuvor mit den Schülerinnen und Schülern die Grundlagen der Wirtschaftskunde sowie Grundsätze einer nachhaltigen Entwicklung mit dem Fokus nachhaltiges Wirtschaften erarbeitet und den Vorbereitungstext gemeinsam gelesen und interaktiv unter Berücksichtigung der unterschiedlichen Bedürfnisse

\footnotetext{
Hierbei sei angemerkt, dass die Testung im Medienraum der Schule durchgeführt wurde, welcher neben einem interaktiven Whiteboard und einem Beamer ebenso eine Video- und Tonanlage beinhaltet. Den Schüler/innen ist bekannt, dass die Unterrichtseinheiten im Medienraum aufgezeichnet werden und es wurden die schriftlichen Einverständniserklärungen der Eltern eingeholt.

3 Diese Informationen wurden im Rahmen der Vorbereitung durch den Klassenlehrer an die zwei Autorinnen weitergegeben.

4 An dieser Stelle sei Katharina Lanzmaier-Ugri gedankt, welche bei der Auswahl der ungarischen Lehrmaterialien als Übersetzerin fungierte.
}

von Frauen und Männern in ihren unterschiedlichen Lebenslagen in Kleingruppen diskutiert.

Außerdem hatte er bereits die Gruppeneinteilung für das Rollenspiel vorweg übernommen, um eine ausgeglichene Verteilung der Schüler/innen nach Geschlecht und Deutschkenntnissen pro Gruppe sicherzustellen.

Der Klassenraum wurde vor der Unterrichtseinheit in zwei Hälften eingeteilt. In der vorderen Hälfte wurde ein halber Sitzkreis mit Blick auf das Whiteboard eingerichtet. In der hinteren Hälfte des Raumes wurden fünfTischinseln mit je vier Stühlen aufgestellt.

Als Materialien wurden die Kopiervorlagen für die Rollenbeschreibungen als auch die Leitfragen für die Interviews ausgedruckt.

Als Einstieg für die Unterrichtseinheit wurden zwei Folien zum Thema ,Nachhaltiger Konsum' gezeigt, um zuvor Gelerntes zu wiederholen. Auf den Folien waren die Begriffe Nachhaltigkeit, Gesellschaft, Ökonomie und Ökologie nicht nur auf Deutsch, sondern auch in den Sprachen Ungarisch und Arabisch eingeblendet, da diese die von den Schülerinnen und Schülern mit Migrations- oder Fluchthintergrund die mehrheitlich gebrauchten Erstsprachen darstellen.

Beim Einblenden der Begriffe in verschiedenen Sprachen zeigten die Schüler/innen sich überrascht und aufmerksam. Gemeinsam wurden die Begrifflichkeiten auf Anweisung der Erstsprachler/innen von allen ausgesprochen und durch die Mitarbeit von weiteren Schülerinnen und Schülern auch in die Sprachen Kurdisch, Farsi, Englisch und Französisch übersetzt.

Nach dieser kurzen Einführung wurden die vier Rollen kurz vorgestellt und es folgte die Anweisung des Klassenlehrers für die Gruppenaufteilung. Ohne Zutun der Lernenden wurde der ungarische Schüler ohne Deutschkenntnisse von den Schülerinnen und Schülern selbst automatisch einer Gruppe zugeordnet.

Bei der Aufteilung der Rollen fiel auf, dass bestimmte Schüler/innen sich sogleich für bestimmte Rollen freiwillig meldeten. So war unter einem Schüler mit Fluchthintergrund die Rolle des unbegleiteten minderjährigen Flüchtlings sehr beliebt, da dieser angab zu wissen, „wie so ein Heim läuft“; die Rolle der Geschäftsinhaberin wurde speziell von mehreren Mädchen präferiert.

Nach der Aufteilung der Gruppen und Rollen teilte sich die Klasse auf die bereits vor Unterrichtsbeginn vorbereiteten Tischinsel auf und die Schüler/innen lasen sich individuell in die ihnen zugeteilten Rollen ein. Im Anschluss begann die Durchführung der Interviews nach dem Prinzip des oben beschriebenen ,Heißen Stubls.

Die Schüler/innen orientierten sich bei ihren Interviewfragen an den zuvor ausgeteilten Leitfragen, fragten aber dennoch nach, um Details der jeweiligen Lebenssituation besser zu verstehen. 
Während der Interviewdurchführung, bei welcher der ungarische Mitschüler auf Grund der fehlenden Deutschkenntnisse nicht folgen konnte, bearbeitete er das zuvor vorbereitete Arbeitsblatt, ohne dabei aber den Gruppentisch zu verlassen. Auf dem Arbeitsblatt war auf Ungarisch die Instruktion angegeben, dass die Ergebnisse des Arbeitsblattes, sofern vom Schüler gewünscht, am Ende der Unterrichtseinheit von ihm auf Ungarisch präsentiert werden könnten, um den anderen Schülerinnen und Schülern einen Einblick in die ungarische Sprache zu geben.

\section{Abschluss}

Nach Ablauf aller vier Interviews nahm die gesamte Klasse wieder im vorderen Teil des Raumes Platz und es wurden die in den Interviewsettings erarbeiteten Lösungsvorschläge gesammelt und auf einem White Board dokumentiert. Hierbei wurde darauf geachtet, Schülerinnen wie auch Schüler in gleichem Ausmaß in die Ideensammlung einzubinden, Fragen und Diskussion willkommen zu heißen und gleichberechtigt wertzuschätzen.

Bei der Sammlung der Lösungen diskutierten die Schüler/innen angeregt, welche Lösungen für welche Rollen anwendbar wären und welche nicht. So wurde von den Schülerinnen und Schülern erkannt, dass der Kauf von Bio-Fleisch für die Rolle des unbegleiteten minderjährigen Asylwerbers aus finanziellen Gründen wahrscheinlich nicht möglich ist und der alleinerziehende Vater für den Transport der Kinder und der Einkäufe schwer auf sein Auto verzichten könne. Den Schülerinnen und Schülern wurde damit klar, wie unterschiedliche Bedürfnisse aus unterschiedlichen Perspektiven und Lebensumständen hervorgehen können. Die Klasse einigte sich schlussendlich darauf, dass die Benutzung eines Autos in Ordnung ist, wenn es sich nicht anders vermeiden lässt, aber das der Verzicht von Fleisch generell von allen, unabhängig vom Einkommen, möglich ist. Auch wurden Lösungen genannt, die für alle Rollen durchführbar sind, wie das Ausschalten des Lichts am Tag und ein bewusster und erschwinglicher Einkauf von saisonalen Lebensmitteln bei lokalen Bauernmärkten in der Stadt, die den Schülerinnen und Schülern bekannt sind. Kreative und gemeinschaftlich-orientierte Lösungsansätze wie beispielsweise Gemeinschaftsgärten, Car-Sharing oder Kleidertausch wurden von den Schülerinnen und Schülern nicht genannt, könnten aber ebenso der Klasse vorgestellt werden, um weitere Lösungen zu entwickeln.

Um auch wieder den Ungarisch sprechenden Schüler einzubinden, wurden die letzten fünf Minuten der Unterrichtseinheit dazu genutzt, ihn die Ergebnisse der Bearbeitung seines Arbeitsblattes präsentieren zu lassen. Er war durchaus dazu bereit und sein Vortrag führte $\mathrm{zu}$ allgemeinem Erstaunen und vereinzeltem Gelächter, welches aber sofort durch den Klassenlehrer unterbunden wurde. Den Schülerinnen und Schülern wurde daraufhin erklärt, dass der Sinn dieser Übung ist, zu verstehen wie es sich anfühlt, einen Vortrag zu hören, bei dem man kein Wort versteht. Weiters wurde darauf hingewiesen, dass manche von ihnen sicher auch die Erfahrung gemacht haben, wie sich diese spezielle Situation anfühlt. Im Anschluss an diese Erklärung entschuldigten sich die Schüler/innen, die zuvor gelacht hatten und hörten den Vortrag aufmerksam zu Ende.

Die Unterrichtseinheit endete mit der Anweisung, die gemeinsam erarbeiteten Lösungen selbst noch einmal nieder zu schreiben, um diese in der nächsten Einheit noch einmal gemeinsam zu wiederholen.

\section{$7 \quad$ Reflexion und Fazit}

Generell kann festgestellt werden, dass die Klasse die Unterrichtseinheit sehr positiv aufnahm. Die Schüler/ innen arbeiteten interessiert und aktiv an den Aufgabenstellungen mit, nahmen die Methoden an und arbeiteten selbstständig in den Gruppen. Trotz des engen Zeitplans konnten alle Arbeitsschritte durchgeführt werden, auch wenn insgesamt mehr Zeit für die Interviewsituation, Heißer Stubl' in Anspruch genommen werden hätte können. Das Klassenklima war von einer insgesamt sehr neugierigen, offenen und positiven Stimmung geprägt.

Auffallend war, dass die Rollen von den Schülerinnen und Schülern ohne Nachfragen akzeptiert wurden. Es gab keinerlei Verwunderung über die nicht dem Mainstream folgenden Lebens- und Familiensituationen der Rollen. Im Gegenteil nahmen die Schüler/innen die Lebensbeschreibungen interessiert an bzw. es fanden sich einige, wie bereits beschrieben, in den unterschiedlichsten Rollen wieder. Hier wurde deutlich, dass die soziale und kulturelle Diversität für die Schüler/innen eine Lebensrealität darstellt, die sie selbstverständlich auch in den Lehrmaterialien akzeptierten. Ein positives Lernerlebnis für die Schüler/ innen war in diesem Kontext, ihre eigene Diversität in den Lehrmaterialien wieder zu finden. Besonders auffällig war dies bei den Schülerinnen und Schülern mit Fluchthintergrund, die sich stark mit der Rolle des afghanischen Asylwerbers identifizieren konnten bzw. bei auch der einzigen Schülerin mit schwarzer Hautfarbe, die sich stark mit der Rolle der Unternehmerin identifizierte, die anhand eines free-licenced Fotos als schwarze Frau abgebildet war. Die nichtstereotypen Geschlechterrollen, wie beispielsweise die des Alleinerziehers oder der lesbischen Studentin der 
technischen Chemie, wurden von den Schülerinnen und Schülern aktiv wahrgenommen, aber nicht hinterfragt und sie reagierten keinesfalls verwundert oder mit Unverständnis.

Ein weiteres positives Lernerlebnis für die Schüler/ innen war der Fokus auf den Wert der vorhandenen Mehrsprachigkeit der Klasse. Die Schüler/innen zeigten sich sehr stolz, Begriffe in ihren nicht-deutschen Erstsprachen den anderen Mitschülerinnen und Mitschülern beizubringen und der ungarische Mitschüler nahm freiwillig die Möglichkeit wahr, seinen Klassenkolleginnen und Klassenkollegen auf Ungarisch ein Kurzreferat vorzutragen. Die Vielfalt der Sprachen wurde auf diese Weise nicht als Barriere, sondern als Mehrwert für den Unterricht gesehen.

Für den Umgang mit der hohen Diversität in der Testklasse bedurfte es einem höheren Ausmaß an Vorbereitung. Fraglich ist hierbei, wie dieser Mehraufwand auch im regulären Unterricht aufgebracht werden kann, wobei dieser Beitrag hierfür eine Hilfestellung darstellen soll.

Insgesamt lässt sich feststellen, dass Aspekte der Diversität in der Durchführung der Einheit und in der Diskussion mit den Schülerinnen und Schülern Aspekte der Geschlechterungleichheit überlagerten. Durch die Anwendung einer gendergerechten Sprache, der Schaffung eines inklusiven Lernklimas, der Ermunterung von Schülerinnen und Schülern sich bewusst zu Wort zu melden, die Aufteilung der Gruppen in gendergerechte Gruppen und die selbstverständliche Vermittlung davon, dass Frauen und Mädchen gleichberechtigte Rollen in der Gesellschaft einnehmen, wurde Geschlechtergerechtigkeit als selbstverständlich angenommen. Dadurch konnte ein Fokus auf die intersektionale Verknüpfung von sozialen Verhältnissen und deren Diversität gelegt werden.

Die von den Schülerinnen und Schülern erarbeitete Lösungen weisen darauf hin, dass die Schüler/innen eine Sensibilisierung für einen nachhaltigen Konsum erfahren haben, selbstständig Ideen für nachhaltiges Konsumverhalten erarbeiten können und diese in den Kontext von diversen Lebenssituation von Frauen und Männern stellen sowie anpassen können. Die Unterrichtseinheit war demnach ein gelungener Versuch, das ganzheitliche Verständnis für eine nachhaltige Entwicklung zu fördern.

\section{Dank und Förderhinweis}

\section{Publiziert mit Unterstützung der Universität Graz}

KARL-FRANZENS-UNIVERSITÄT GRAZ UNIVERSITY OF GRAZ

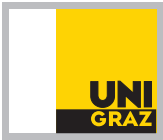

\section{Literatur}

Althans, B. (2016): The desire to consume: Ernährung zwischen Shoppen, Kochen und Verzehren als Inszenierung weiblicher Geschlechtsidentität? In: Althans, B. \& J. Bilstein (Hrsg.): Essen - Bildung - Konsum. Pädagogisch-anthropologische Perspektiven. Wiesbaden: Springer, 225-244.

Bauhardt, C. (2010): Ökologiekritik. Das Mensch-NaturVerhältnis aus der Geschlechterperspektive. In: Becker, R. \& B. Kortendiek (Hrsg.): Handbuch Frauen- und Geschlechterforschung. Theorie, Methoden, Empirie. 3. Erweiterte und durchgesehene Auflage. Wiesbaden: VS Verlag, 322-327.

Bundesministerium für Bildung (2014): Grundsatzerlass zum Unterrichtsprinzip „Erziehung zur Gleichstellung von Frauen und Männern“. Geschäftszahl: 15.510/60Präs.3/95, Wien. https://www.bmb.gv.at/ministerium/ rs/1995_77.html (23.10.2017).

Bundesministerium für Bildung (2016): Bundesgesetzblatt für die Republik Österreich. 219. Änderung der Verordnung über die Lehrpläne der allgemein bildenden höheren Schulen; Änderung der Bekanntmachung der Lehrpläne für den Religionsunterricht an diesen Schulen. Teil II, Wien. https://www.ris.bka.gv.at/Dokumente/BgblAuth/BGBLA_2016_II_219/BGBLA_2016_II_219. pdf (23.10.2017).

Bundesministerium für Bildung (2017): Erziehung zur Gleichstellung von Frauen und Männern, Wien. https:// www.bmb.gv.at/schulen/unterricht/prinz/erziehung gleichstellung.html (23.10.2017).

Bundesministerium für Unterricht, Kunst und Kultur (2008): Gender Kompetenz und Gender Mainstreaming. Kriterienkatalog für Schulen. Wien. http://www.eduhi. at/dl/gekos-kriterien-online.pdf (23.10.2017).

Eder-Kornfeld, R. (2014): Konsum und Gesellschaft. Tipps für einen nachhaltigen Lebensstil. In: Wiener Zeitung, 13.01.2014. http://www.wienerzeitung.at/themen_channel/wirtschaftsservice/konsum_und_gesellschaft/599823_Tipps-fuer-einen-nachhaltigen-Lebensstil.html (23.10.2017).

Fridrich, C. (2014): Von der Theorie zur Praxis: lebensweltorientierte ökonomische Bildung im Unterrichtsalltag. In: GEOGRAZ, Grazer Mitteilungen der Geographie und Raumforschung 54, 16-25.

Fridrich, C. (2016): Basiskonzepte in Geographie und Wirtschaftskunde - ein Vorschlag für die Sekundarstufe I. In: GEOGRAZ. Grazer Mitteilungen der Geographie und Raumforschung 59, 24-31.

Haarmann, M.-P. (2014): Sozioökonomische Bildung unter der Zielperspektive der gesellschaftlichen Mündigkeit. In: Fischer, A. \& B. Zurstrassen (Hrsg.): Sozioökonomische Bildung. Bonn: Bundeszentrale für politische Bildung, 206-222.

Hörmann K., E. Koblmiller \& L. Tröscher (2017): (De-)Konstruktion des klassischen Familienbildes im politisch bildenden GW-Unterricht. In: GW-Unterricht 145, 42-52. https:// dx.doi.org/10.1553/gw-unterricht145s42 (23.10.2017). 
Koyama, E. \& Weasel, L. (2003): From Social Construction to Social Justice. Transforming. How We Teach About Intersexuality. In: Intersex Initiative Portland (ed.): Teaching Intersex Issues. A Guide for Teachers in Women's, Gender \& QueerStudies. TheSecondEdition.PortlandOregon.http:// eminism.org/store/pdf-zn/ipdx-teacher.pdf (23.10.2017)

Lackner, E. (2012): Heißer Stuhl. In: Universität für Bodenkultur Wien, Karl-Franzens-Universität Graz \& Technische Universität Graz (Hrsg.): Sustainicum Collection. Lehrmaterialien für Bildung zur Nachhaltigkeit. http://www.sustainicum.at/de/tmethods/view/24.HeierStuhl (23.10.2017).

Mehren, M., R. Mehren, U. Ohl \& C. Resenberger (2015): Die doppelte Komplexität geographischer Themen: Eine lohnenswerte Herausforderung für Schüler und Lehrer. In: Geographie aktuell \& Schule, 37, 4-11.

Münst, A. S. (2008): Intersektionalität als Perspektive der Migration. In: POLITIC 1, 41-54.
Wastl-Walter, D. (2010): Gender Geographien. Geschlecht und Raum als soziale Konstruktion. Sozialgeographie kompakt, Band 2. Stuttgart: Franz Steiner Verlag.

Winkler, G. \& N. Degele (2009): Intersektionalität. Zur Analyse sozialer Ungleichheiten. Bielefeld: transcript.

Zimmermann, F. M. (2016a): Globale Herausforderungen und die Notwendigkeit umzudenken- wie soll das funktionieren? In: ebenda (Hrsg.): Nachhaltigkeit wofür? Von Chancen und Herausforderungen für eine nachhaltige Zukunft. Berlin, Heidelberg: Springer, 25-58.

Zimmermann, F. M. (2016b): Was ist Nachhaltigkeit - eine Perspektivenfrage? In: ebenda (Hrsg.): Nachhaltigkeit wofür? Von Chancen und Herausforderungen für eine nachhaltige Zukunft. Berlin, Heidelberg: Springer, 1-24. 


\section{Anhang}

Rollenbeschreibungen: Amir, Lisa, Peter und Sarah sind vier fiktive Charaktere mit unterschiedlichen Lebensgeschichten. Doch sie haben vieles gemeinsam: Jede/r von ihnen kann sich an der Gestaltung einer nachhaltigen Zukunft beteiligen!

\section{Rolle 1: Amir, 17 Jahre alt}

Amir lebt seit eineinhalb Jahren in Österreich. Er kommt ursprünglich aus Afghanistan und wohnt mit 40 anderen jugendlichen Geflüchteten in einem Heim in einer österreichischen Kleinstadt. Amirs Mutter und seine zwei kleinen Brüder leben in Afghanistan. Seine ältere Schwester und sein Vater sind bei einem Angriff der Taliban gestorben. Amir teilt sich sein Zimmer mit Mohammad und Ali aus Syrien und Farid aus Afghanistan. Amir bekommt im Monat von der Heimleitung 110 Euro Essensgeld und 40 Euro Taschengeld ausbezahlt. Damit kauft er Lebensmittel beim Supermarktdiskonter und in einem türkischen Spezialgeschäft. Seine Mahlzeiten bereitet er in der Gemeinschaftsküche zu. Am Vormittag besucht er einen Deutschkurs und am Abend spielt er am liebsten mit seinen Mitbewohnern Fußball. Amir versteht sich gut mit seinen Mitbewohnern, nur manchmal gibt es Streitigkeiten, wenn sich jemand nicht an den Putzplan hält. Amir macht sich Sorgen um seine Familie in Afghanistan und möchte so rasch wie möglich seinen Hauptschulabschluss nachholen und eine Arbeit als Programmierer finden, damit er sich um seine Familie kümmern kann.

\section{Rolle 3: Peter, 39 Jahre alt}

Peter wohnt mit seinen zwei Kindern Max, 3 Jahre alt, und Leonie, 7 Jahre alt, in einer gemütlichen drei-Zimmer-Wohnung in einer mittelgroßen Stadt in Österreich. Peter ist seit zwei Jahren geschieden und arbeitet 40 Stunden in der Woche als mittlerer Beamter für die Stadtverwaltung, wo er ein Einkommen von 1650 Euro netto pro Monat bezieht. Jeden Morgen bringt er mit dem Auto Max und Leonie in den Kindergarten und zur Schule und holt sie nach seiner Arbeit am Nachmittag ab. Peter ist sehr froh, dass es im Kindergarten und in der Schule eine Nachmittagsbetreuung für seine Kinder gibt. Am frühen Abend erledigt er noch rasch Einkäufe, kocht das Abendessen und kümmert sich um den Haushalt. In seiner Freizeit geht er am liebsten mit seinen Kindern auf den Spielplatz, wenn diese nicht - wie jedes zweite Wochenende - bei ihrer Mutter sind.

\section{Rolle 2: Lisa, 20 Jahre alt}

Lisa studiert seit einem Jahr technische Chemie und ist deswegen aus ihrem Heimatdorf im Süden der Steiermark in eine mittelgroße Stadt in Österreich gezogen. Sie teilt sich mit Andrea, die Erziehungswissenschaften studiert, und Robert, der Geographie studiert, eine Wohngemeinschaft, in welcher jede/r von ihnen ein eigenes Zimmer hat. Mit ihrem Fahrrad braucht Lisa nur 15 Minuten von ihrer Wohnung zum Uni-Campus.

Da Lisas Eltern über ein nicht so hohes Einkommen verfügen, bekommt Lisa ein Stipendium in der Höhe von 680 Euro pro Monat. Dafür bezahlt sie die Miete in der Höhe von 280 Euro im Monat, das Essen in der Uni-Mensa und was sie sonst für ihren Studienalltag benötigt. Ihre Freizeit verbringt Lisa am liebsten mit ihrer Freundin Anja. Nächsten Monat feiern die beiden den ersten Jahrestag ihrer Beziehung.

\section{Rolle 4: Sarah, 55 Jahre alt}

Sarah betreibt in einer österreichischen Kleinstadt seit fast zwanzig Jahren ein angesehenes Computerfachgeschäft. Sie ist ausgebildete Marketingexpertin und verkauft in ihrem Laden IT-Zubehör und ausgewählte Computerhardware. Sarah beschäftigt insgesamt fünf Mitarbeiter/-innen und trinkł bei einem ausführlichen Beratungsgespräch mit ihren Kundinnen und Kunden gerne einen guten Kaffee. Ihre große Dachgeschosswohnung befindet sich in einer Altbauwohnung gegenüber von ihrem Geschäft. Dort wohnt sie mit ihrem 40-jährigen Lebensgefährten Gerhard, der sich in der Stadtpolitik engagiert. In ihrer Freizeit machen die beiden am liebsten Ausflüge in ihrem Cabriolet und teilen sich ihre Leidenschaft für gute Restaurants und ausgefallene Weine. Sarah hat pro Monat nach Abzug aller Spesen für ihr Geschäft rund 4000 Euro für sich zur freien Verfügung. 


\section{Arbeitsauftrag}

\section{Aufgabenstellung I}

- Lies deine Rollenbeschreibung!

- Versetze dich in deine Rolle: Wer bist du, was machst du, was ist dir wichtig in deinem Leben?

- Überlege was du tun kannst, um nachhaltiger zu leben!

- Einkaufen

- Essen und Trinken

- Heizen

- Mobilität

- Verwendung von Elektrogeräten

- Kleidung

$-\quad \cdots$

\section{Aufgabenstellung II}

- Setze dich auf den ,Heißen Stuhl' und stelle dich deiner Gruppe vor (Name, Alter, Beruf, Einkommen, Wohnsituation, Beziehungsstatus)!

- Stelle dich den Fragen deiner Mitschüler/innen!

- Was machst du an einem typischen Wochentag in deinem Leben?

- Was ist dir wichtig?

- Was brauchst du, um glücklich zu sein?

- Worauf möchtest du nicht verzichten?

- Was könntest du in deinem Leben ändern, um nachhaltiger zu sein?

$-\quad \cdots$ 